\title{
Dual-Objective NMPC: Considering Economic Costs near Manifolds
}

\author{
Niels van Duijkeren, Timm Faulwasser, and Goele Pipeleers
}

\begin{abstract}
This paper presents a dual-objective Nonlinear Model Predictive Control (NMPC) algorithm for stabilizing a target neighborhood of a state space manifold of a nonlinear dynamical system and for concurrently optimizing an economic objective in this neighborhood. The control design is based on the transverse normal form description of the system dynamics. The NMPC scheme solves two Optimal Control Problems (OCPs) in sequence, the first (transversal) OCP provides conditions for convergence to the target neighborhood. The second (tangential) OCP optimizes the economic objective without compromising convergence. Stability and performance properties of the resulting control scheme are discussed and its efficacy is illustrated in a tutorial example.
\end{abstract}

Index Terms-Nonlinear model predictive control, manifold stabilization, transverse normal forms, economic NMPC.

\section{INTRODUCTION}

$\mathbf{I}$ $\mathrm{N}$ many control applications the desired closed-loop behavior comprises a mix of objectives: certain outputs should converge to a reference, while others ought to perform optimally according to an economic performance criterion. For instance, an autonomous driving car following the lane center while optimizing a trade-off between driver comfort, fuel economy and travel time; or traversing a robotic manipulator along a neighborhood of a reference path while minimizing the transition time.

Transverse feedback linearization refers to the concept of finding a feedback equivalent description of a system that is linear in the directions transverse to the manifold $[2,3]$. It allows decoupling the stabilization of a given manifold from controlling the motion on the manifold. It is used for specific manifold control problems such as path following, where a one-dimensional output manifold is translated into a higherdimensional state-space manifold [4]. Predictive manifoldstabilization approaches have been used together with Transverse Normal Forms (TNFs): in [5] TNFs serve as a tool to design stabilizing terminal sets for model predictive pathfollowing control, and [6] proposes a sequential predictive control approach.

Concurrently, in the realm of Nonlinear Model Predictive Control (NMPC), the developments on economic NMPC

N. van Duijkeren and G. Pipeleers are with the Department of Mechanical Engineering of the KU Leuven and the DMMS lab of Flanders Make, Celestijnenlaan 300, B-3001, Belgium (e-mail: \{niels.vanduijkeren, goele.pipeleers\}@kuleuven.be).

T. Faulwasser is with the Institute for Automation and Applied Computer Science, Karlsruhe Institute of Technology, Eggenstein-Leopoldshafen D76344, Germany (e-mail: timm.faulwasser@ieee.org).

A preliminary version of this manuscript was presented at the 56th IEEE Conference on Decision and Control [1]. suggest to consider quite general objectives that are not directly related to stabilization [7, 8]. Compared to the usual tracking formulations, closed-loop stability, convergence, and performance are often more difficult to address in economic NMPC. Recent progress includes [7, 8]: dissipativity-based methods employing a stabilizing terminal-constraint [9, 10]; and approaches based on dissipativity and turnpike theory that do not require terminal constraints [11, 12]. However, the progress on economic NMPC raises the question whether dual objectives of economic and stability requirements can be approached more directly, cf. [13, 14].

The present paper addresses a specific case of dual objectives; i.e. it investigates the question of how to consider practical stabilization of a given reference manifold in the state space while optimizing an economic criterion near this manifold. Put differently, herein a sequential approach for dual-objective NMPC applied to transverse feedback linearizable systems is proposed. The main contribution is that the proposed method allows to explicitly state the output manifold that is to be stabilized, while leaving freedom to independently formulate a desired economic objective. Furthermore, the method enables to unambiguously control the maximum allowed deviation from the manifold to benefit economic performance. To the best of the authors' knowledge, this combination of featureswhich is attractive for motion control and mechatronics-has not been addressed before.

Similar to [6], it is suggested to solve two OCPs sequentially at every sampling instant. First an OCP is solved to nominally stabilize the target manifold. Subsequently, the solution of the first OCP is used to establish convergence conditions for a neighborhood of the manifold. These conditions are then imposed in the second OCP that optimizes the motion on the manifold. Extending the results of [6], economic objectives on the target manifold can be explicitly accounted for. Furthermore, by enforcing convergence to an ellipsoidal neighborhood of the manifold, whose radius is a design parameter-i.e. a tuning knob-economic performance can be improved by deviating slightly from the target manifold. Quantification of the closed-loop economic NMPC performance with respect to the infinite horizon OCP [15] is in general difficult and beyond the scope of this paper. However, in a numerical case-study it is observed that the closed-loop performance is highly correlated to the size of the neighborhood. Although the proposed method relies on TNFs as a technical vehicle in the control design phase, it is shown that they need not to be used in the implementation. This can be particularly useful when the OCP in the original state space is numerically better conditioned for receding-horizon optimization compared 
to TNF coordinates.

The goals and scope of the present paper are closely in line with the preliminary conference paper [1]. Both apply a sequential approach to dual-objective NMPC to stabilize a target neighborhood of a manifold while optimizing economic costs for the motion tangent to the manifold. However, they significantly differ in terms of how the solution of the first OCP is used to establish convergence conditions for the second OCP. The advantages of the present contribution over [1] include reduced conservatism of the convergence condition and allowing the OCPs to be solved in the original state-space coordinates.

The remainder of the paper is organized as follows. Section II formally states the problem addressed by the presented NMPC algorithm. Section III briefly recapitulates transverse feedback linearization, on which the predictive control scheme is then introduced. Section IV discusses recursive feasibility and convergence properties of the proposed control scheme. Section $\mathrm{V}$ discusses an implementation approach to the solution method. In Section VI the results of a numerical casestudy are discussed. Finally, Section VII draws the main conclusions of the paper.

\section{Notation}

Lower case symbols, $x, y, u$, etc., are used for scalar and vector valued variables, placeholder notation $\bullet(\cdot)$ is used to denote a function. The index-subscript $\bullet_{i}$ is short for $\bullet\left(t_{i}\right)$. The $\boldsymbol{\bullet}, \tilde{\bullet}, \hat{\bullet}$-notation is exclusively used for trajectories and therefore the placeholder notation is often omitted. Moreover, - is reserved for predicted trajectories inside optimal control problems, the diamond and asterisk superscripts (e.g., $\bar{\bullet}^{\diamond}$ ) indicate this function is optimal with respect to a specific optimal control problem. Given an ODE $\dot{x}=f(x, u)$, an initial condition $x_{0}$ and an input $u(\cdot)$, the solution trajectory is denoted as $x\left(\cdot ; x_{0}, u(\cdot)\right)$. Most signals have a finite support which follows from the context. The boundary of a set $\mathcal{N} \subset \mathbb{R}^{n}$ is denoted as $\partial \mathcal{N}$. The distance of $x \in \mathbb{R}^{n}$ to a compact set $\mathcal{N} \subset \mathbb{R}^{n}$ is denoted as $\|x\|_{\mathcal{N}}$.

\section{Problem Statement}

The problem statement involves four ingredients: a system, a target manifold, an economic running cost, and a target tolerance. Firstly, consider nonlinear input-affine systems

$$
\begin{aligned}
& \dot{x}=f(x)+\sum_{i=1}^{m} g_{i}(x) u_{i}=f(x)+G(x) u, x(0)=x_{0} \\
& y=h(x),
\end{aligned}
$$

with states $x \in \mathbb{R}^{n}$, inputs $u \in \mathcal{U} \subset \mathbb{R}^{m}$, and outputs $y \in \mathbb{R}^{p}$, with $p \leq m$ for which the following assumptions hold:

Assumption 1 (Differentiable dynamics). The vector fields $f, g_{1}, \ldots, g_{m}: \mathbb{R}^{n} \rightarrow \mathbb{R}^{n}$ are sufficiently often continuously differentiable.

Assumption 2 (Absolute continuity of trajectories). For any $x_{0} \in \mathbb{R}^{n}$ and any input function $u(\cdot) \in \mathcal{L}^{\infty}\left(\mathbb{R}_{0}^{+}, \mathcal{U}\right)$, the solution trajectories of system (1) are absolutely continuous.
Secondly, consider the following target manifold

$$
\mathcal{M}=\left\{x \in \mathbb{R}^{n} \mid \sigma(h(x))=0\right\} \subset \mathbb{R}^{n},
$$

with $\sigma: \mathbb{R}^{p} \rightarrow \mathbb{R}^{l}, l \leq p \cdot{ }^{1}$ For ease of notation $\alpha:=\sigma \circ h$ is introduced. Since $\mathcal{M}$ is generally not controlled invariant, let $\mathcal{M}^{\star}$ denote the maximum controlled invariant subset contained in $\mathcal{M}$, rendered invariant by some $u \in \mathcal{U}$.

Assumption 3 (Feasibility of manifold stabilization). The maximal controlled invariant subset of $\mathcal{M}$, denoted by $\mathcal{M}^{\star} \subseteq$ $\mathbb{R}^{n}$, is a non-empty, closed embedded submanifold of the state space. The dimension of $\mathcal{M}^{\star}$ is denoted by $n^{\star}$.

Assumption 3 is a basic feasibility requirement for the manifold stabilization problem, meaning that if $x_{0} \in \mathcal{M}^{\star}$ there exists an input $u(\cdot) \in \mathcal{U}$ such that $x\left(t ; x_{0}, u(\cdot)\right) \in \mathcal{M}^{\star}, \forall t \geq$ $t_{0}$.

As a third ingredient, the user formulates an economic running cost function $F^{\text {eco }}: \mathbb{R}^{n} \times \mathbb{R}^{m} \rightarrow \mathbb{R}$ which, when minimized, leads to the optimal motion on the manifold.

Finally, the target tolerance $\varepsilon \in \mathbb{R}_{0}^{+}$controls the size of a target neighborhood around the manifold and is crucial in the problem statement:

Problem 1 (Economic operation near a target manifold). Given system (1), the manifold (2), the economic running cost $F^{e c o}$, and the target tolerance $\varepsilon$, design a controller that achieves:

(a) Manifold convergence: The state $x$ converges to an $\varepsilon$ neighborhood of the target submanifold $\mathcal{M}^{\star}$,

$$
\lim _{t \rightarrow \infty}\|\sigma(h(x(t)))\|_{2} \leq \varepsilon
$$

(b) Economic performance near manifold: Implement an economic NMPC with objective $J^{\text {eco }}=$ $\int_{t_{i}}^{t_{i}+T} F^{e c o}(x(\tau), u(\tau)) \mathrm{d} \tau$ for some $T$ describing optimal near-manifold motion, and enables monotonic improvement of $J^{\text {eco }}$ for increasing $\varepsilon$.

(c) Constraint satisfaction: The constraints on the inputs $u(t) \in \mathcal{U}$ are satisfied at all times.

The above problem is generally complex. One might try to tackle it by an offline optimization phase, accompanied by an online reference tracking phase. Alternatively, one could devise a switched control scheme, with a mode for manifold stabilization and a mode for economic control near the manifold. In the next section, a single-mode online control strategy is introduced that realizes both manifold stabilization and economic control, and requires no offline generated reference trajectories for the states and inputs.

\section{SOlution ApproACH}

A sampled-data NMPC control approach is adopted with fixed sampling intervals of length $\delta \in \mathbb{R}^{+}$. Two subsequent OCPs are solved in order to compute the closed-loop input signal applied to the system. The OCPs are defined using a suitable transverse normal form that is recalled next.

\footnotetext{
${ }^{1}$ For example, an implicit parameterization of a reference path in the output space typically leads to $l<p$.
} 


\section{A. Transverse Normal Forms}

Assumption 4 (Vector relative degree). For $\alpha(x)=$ $\left[\alpha_{1}(x) \cdots \alpha_{l}(x)\right]^{\top}, \alpha_{1}(x), \ldots, \alpha_{l}(x): \mathbb{R}^{n} \rightarrow \mathbb{R}^{l}$, it holds that

(a) $\mathcal{M}^{\star} \subset\left\{x \in \mathbb{R}^{n} \mid \alpha_{1}(x)=\ldots=\alpha_{l}(x)=0\right\}$, and

(b) $\alpha(x)$ yields a well-defined vector relative degree $\rho=$ $\left\{\rho_{1}, \ldots, \rho_{l}\right\}$ on $\mathbb{R}^{n}$ with $\sum_{i=1}^{l} \rho_{i}=n-n^{\star}, c f .[16,17]$.

The authors of [3, Thm. 3.1] show that if Assumptions 3 and 4 hold, then around any point of $\mathcal{M}^{\star}$ there exists a local diffeomorphism $\Phi: \mathbb{R}^{n} \rightarrow \mathbb{R}^{n-n^{\star}} \times \mathbb{R}^{n^{\star}}, x \mapsto(\xi, \eta)$, and a transversely linearizing feedback

$$
u=a(\xi, \eta)+b(\xi, \eta)\left[v^{\top} w^{\top}\right]^{\top}=: \kappa(\xi, \eta, v, w),
$$

such that, in the $(\xi, \eta)$-coordinates, (1a) subject to (3) reads

$$
\begin{aligned}
& \dot{\xi}=A \xi+B v \\
& \dot{\eta}=f^{0}(\xi, \eta)+G^{\pitchfork}(\xi, \eta) v+G^{\|}(\xi, \eta) w,
\end{aligned}
$$

with the pair $(A, B)$ controllable and the image $\Phi\left(\mathcal{M}^{\star}\right)=$ $\{(\xi, \eta) \mid \xi=0\}$. The transverse dynamics are described by (4a) that resembles a set of $l$ integrator chains, with $\xi=\left[\alpha_{1} \cdots \alpha_{1}^{\left(\rho_{1}-1\right)} \cdots \alpha_{l} \cdots \alpha_{l}^{\left(\rho_{l}-1\right)}\right]^{\top} \in \mathbb{R}^{n-n^{\star}}$ and $v=$ $\left[\alpha_{1}^{\left(\rho_{1}\right)} \cdots \alpha_{l}^{\left(\rho_{l}\right)}\right]^{\top} \in \mathbb{R}^{l}$. The tangential dynamics, with state $\eta \in \mathbb{R}^{n^{\star}}$ and the tangential input $w \in \mathbb{R}^{m-l}$ are governed by (4b). This generally nonlinear subsystem describes the motion on $\mathcal{M}^{\star}$.

Remark 1. Since $\Phi$ is a diffeomorphism, the relation between $(\xi, \eta)$ and $x$ is locally bijective and invertible. To denote the dependency of a function on $\xi$, $\eta$ or both, $x$ is often used for brevity. For example, $f(\xi, \eta)=f(\Phi(x))=: f(x)$.

\section{B. Transverse $O C P$}

The cost functional for the first OCP is defined as,

$$
J^{\pitchfork}(\hat{\xi}, \hat{v})=\int_{0}^{T^{\pitchfork}} F^{\pitchfork}(\hat{\xi}(\tau), \hat{v}(\tau)) \mathrm{d} \tau+E\left(\hat{\xi}\left(T^{\pitchfork}\right)\right) .
$$

Assumption 5 (Positive-definite cost function). The cost function $F^{\pitchfork}$ is specified by positive definite matrices $Q, R \succ 0$ such that

$$
F^{\pitchfork}(\xi, v)=\|\xi\|_{Q}^{2}+\|v\|_{R}^{2} \geq \gamma(\|\xi\|), \quad \forall \xi, v,
$$

where $\gamma$ is of class $\mathcal{K}$ and the terminal penalty $E(\xi)=\|\xi\|_{P}^{2}$, with $P \succ 0$ the solution of the algebraic Riccati equation

$$
A^{\top} P+P A+Q=P B R^{-1} B^{\top} P .
$$

Put differently, (5a) is a manifold stabilizing cost where the terminal penalty corresponds to the infinite horizon $L Q R$ value function. The transverse OCP $\Pi^{\dagger}\left(x_{i}\right)$ at time instant $t_{i}$, with an optimization horizon of length $T^{\pitchfork}$, is defined as:

$$
\begin{aligned}
\min _{\bar{\xi}, \bar{\eta}, \bar{v}, \bar{w}} & J^{\pitchfork}(\bar{\xi}, \bar{v}) \\
\text { s.t. } & \dot{\bar{\xi}}=A \bar{\xi}+B \bar{v} \\
& \dot{\bar{\eta}}=f^{0}(\bar{\xi}, \bar{\eta})+G^{\pitchfork}(\bar{\xi}, \bar{\eta}) \bar{v}+G^{\|}(\bar{\xi}, \bar{\eta}) \bar{w} \\
& {\left[\bar{\xi}(0)^{\top} \bar{\eta}(0)^{\top}\right]^{\top}=\Phi\left(x_{i}\right) } \\
& \kappa(\bar{\xi}, \bar{\eta}, \bar{v}, \bar{w}) \in \mathcal{U} \\
& \bar{\xi}\left(T^{\pitchfork}\right) \in \mathcal{E}_{\xi} \quad \bar{\eta}\left(T^{\pitchfork}\right) \in \mathcal{E}_{\eta} .
\end{aligned}
$$

Denote the optimal trajectories from $\Pi^{\pitchfork}\left(x_{i}\right)$ by the quadruple $\zeta^{\diamond}\left(\cdot ; x_{i}\right)=\left(\bar{\xi}^{\diamond}\left(\cdot ; x_{i}\right), \bar{\eta}^{\diamond}\left(\cdot ; x_{i}\right), \bar{v}^{\diamond}\left(\cdot ; x_{i}\right), \bar{w}^{\diamond}\left(\cdot ; x_{i}\right)\right)$. The terminal constraint sets $\mathcal{E}_{\xi}$ and $\mathcal{E}_{\eta}$ in (6f) are chosen such that they satisfy the following assumption, common in the NMPC literature [18, 19]:

Assumption 6 (Existence of terminal controls). For all $x_{0} \in$ $\Phi^{-1}\left(\mathcal{E}_{\xi} \times \mathcal{E}_{\eta}\right)$, there exist input signals $\hat{v}_{\mathcal{E}} \in \mathcal{L}^{\infty}\left([0, \delta], \mathbb{R}^{l}\right)$ and $\hat{w}_{\mathcal{E}} \in \mathcal{L}^{\infty}\left([0, \delta], \mathbb{R}^{m-l}\right)$, such that for all $\tau \in[0, \delta)$ :

$$
\begin{array}{r}
\kappa\left(\hat{\xi}\left(\tau ; x_{0}, \hat{v}_{\mathcal{E}}\right), \hat{\eta}\left(\tau ; x_{0}, \hat{v}_{\mathcal{E}}, \hat{w}_{\mathcal{E}}\right), \hat{v}_{\mathcal{E}}(\tau), \hat{w}_{\mathcal{E}}(\tau)\right) \in \mathcal{U}, \\
\hat{\xi}\left(\tau ; x_{0}, \hat{v}_{\mathcal{E}}\right) \in \mathcal{E}_{\xi}, \quad \hat{\eta}\left(\tau ; x_{0}, \hat{v}_{\mathcal{E}}, \hat{w}_{\mathcal{E}}\right) \in \mathcal{E}_{\eta} \\
\left.E\left(\hat{\xi}\left(s ; x_{0}, \hat{v}_{\mathcal{E}}\right)\right)\right|_{0} ^{\tau} \leq-\int_{0}^{\tau} F^{\pitchfork}\left(\hat{\xi}\left(s ; x_{0}, \hat{v}_{\mathcal{E}}\right), \hat{v}_{\mathcal{E}}(s)\right) \mathrm{d} s
\end{array}
$$

\section{Tangential $O C P$}

The cost functional according to which the trajectories are refined in favor of economic performance is defined as

$$
J^{\|}(\hat{\xi}, \hat{\eta}, \hat{v}, \hat{w})=\int_{0}^{T^{\|}} F^{\|}(\hat{\xi}(\tau), \hat{\eta}(\tau), \hat{v}(\tau), \hat{w}(\tau)) \mathrm{d} \tau,
$$

with $F^{\|}(\xi, \eta, v, w):=F^{\mathrm{eco}}\left(\Phi^{-1}(\xi, \eta), \kappa(\xi, \eta, v, w)\right)$, the userdefined economic running cost. Given the target tolerance $\varepsilon$, the second OCP must not jeopardize convergence in Problem 1a. Consider the target neighborhood to be a level set of the terminal cost function $E$. Therefore, considering

$$
\mathcal{N}=\left\{\xi \in \mathbb{R}^{n-n^{\star}} \mid E(\xi) \leq \varepsilon_{\mathcal{N}}^{2}\right\}
$$

a suitable $\varepsilon_{\mathcal{N}}$ needs to be found for a given $\varepsilon$ (generally introducing conservatism). Section VI-C comments on this issue for a specific example.

Thus, let $\mathcal{J}$ be the set of states $x$ for which the optimal transverse cost in $\Pi^{\pitchfork}(x)$ is upper bounded by $\bar{\jmath}^{\pitchfork}>0$

$$
\begin{aligned}
\mathcal{J}\left(\bar{\jmath}^{\dagger}\right):=\{ & x \in \mathbb{R}^{n} \mid \exists \hat{v}, \hat{w}, \hat{\xi}(\cdot ; x, \hat{v}), \hat{\eta}(\cdot ; x, \hat{v}, \hat{w}) \text { satisfying: } \\
& \kappa(\hat{\xi}, \hat{\eta}, \hat{v}, \hat{w}) \in \mathcal{U} \\
& J^{\pitchfork}(\hat{\xi}, \hat{\eta}, \hat{v}, \hat{w}) \leq \bar{j}^{\pitchfork} \\
& \left.\hat{\xi}\left(T^{\pitchfork}\right) \in \mathcal{E}_{\xi}, \hat{\eta}\left(T^{\pitchfork}\right) \in \mathcal{E}_{\eta}\right\} .
\end{aligned}
$$

The tangential OCP, $\Pi^{\|}\left(x_{i}, \zeta^{\diamond}\left(\cdot, x_{i}\right)\right)$ at time instant $t_{i}$, with an optimization horizon of length $T^{\|}$, reads:

$$
\begin{aligned}
\min _{\bar{\xi}, \bar{\eta}, \bar{v}, \bar{w}} & J^{\|}(\bar{\xi}, \bar{\eta}, \bar{v}, \bar{w}) \\
\text { s. t. } & \dot{\bar{\xi}}=A \bar{\xi}+B \bar{v} \\
& \dot{\bar{\eta}}=f^{0}(\bar{\xi}, \bar{\eta})+G^{\pitchfork}(\bar{\xi}, \bar{\eta}) \bar{v}+G^{\|}(\bar{\xi}, \bar{\eta}) \bar{w} \\
& {\left[\bar{\xi}(0)^{\top} \bar{\eta}(0)^{\top}\right]^{\top}=\Phi\left(x_{i}\right) } \\
& \kappa(\bar{\xi}, \bar{\eta}, \bar{v}, \bar{w}) \in \mathcal{U} \\
& E(\bar{\xi}(\tau)) \leq \max \left\{\varepsilon_{\mathcal{N}}^{2}, E\left(\tilde{\xi}^{\diamond}(\tau)\right)\right\} \forall \tau \in\left[0, T^{\|}\right] \\
& (\bar{\xi}(\delta), \bar{\eta}(\delta)) \in \mathcal{J}\left(\max \left\{\varepsilon_{\mathcal{N}}^{2}, \tilde{J}^{\pitchfork}\left(x_{i}\right)\right\}\right),
\end{aligned}
$$

where

$$
\begin{aligned}
\tilde{J}^{\dagger}\left(x_{i}\right)= & J^{\pitchfork}\left(\bar{\xi}^{\diamond}\left(\cdot ; x_{i}\right), \bar{v}^{\diamond}\left(\cdot ; x_{i}\right)\right) \\
& -\int_{0}^{\delta} F^{\pitchfork}\left(\bar{\xi}^{\diamond}\left(\tau ; x_{i}\right), \bar{v}^{\diamond}\left(\tau ; x_{i}\right)\right) \mathrm{d} \tau .
\end{aligned}
$$


Denote the optimal trajectories from $\Pi^{\|}\left(x_{i}\right)$ by the quadruple $\zeta^{*}\left(\cdot ; x_{i}\right):=\left(\bar{\xi}^{*}\left(\cdot ; x_{i}\right), \bar{\eta}^{*}\left(\cdot ; x_{i}\right), \bar{v}^{*}\left(\cdot ; x_{i}\right), \bar{w}^{*}\left(\cdot ; x_{i}\right)\right)$. Constraint $(11 \mathrm{~g})$ enforces that at future sampling instances, the value function of $\Pi^{\dagger}$ is bounded such that nominal asymptotic convergence of the closed-loop system can be guaranteed. Intuitively speaking, via $(11 \mathrm{~g})$ a manifold-stabilizing backup trajectory departing from $\bar{x}^{*}\left(\delta ; x_{i}\right)$ is guaranteed to exist. To avoid unnecessarily high computational complexity, constraint $(11 \mathrm{~g})$ is only enforced on the predicted state at the next sampling time instance. For all other points in time, constraint (11f) ensures that $\Pi^{\|}$seeks open-loop optimal trajectories converging to the target neighborhood. Note that the prediction horizons in $\Pi^{\|}\left(x_{i}, \zeta^{\diamond}\left(\cdot, x_{i}\right)\right)$ and $\Pi^{\dagger}\left(x_{i}\right)-T^{\|}$in (8) and $T^{\dagger}$ in (5a) - need not to be identical. Thus, in order to obtain appropriate support, the trajectory $\tilde{\xi}^{\diamond}$ is either a truncation of $\bar{\xi}^{\diamond}$ or an extension computed using a terminal control law for $\mathcal{E}_{\xi}$, for instance the optimal infinite-horizon LQR trajectory according to the $Q$ and $R$ matrices in (5) for system (4a).

The sequential approach to the Dual-Objective Economic NMPC problem is summarized in Algorithm 1:

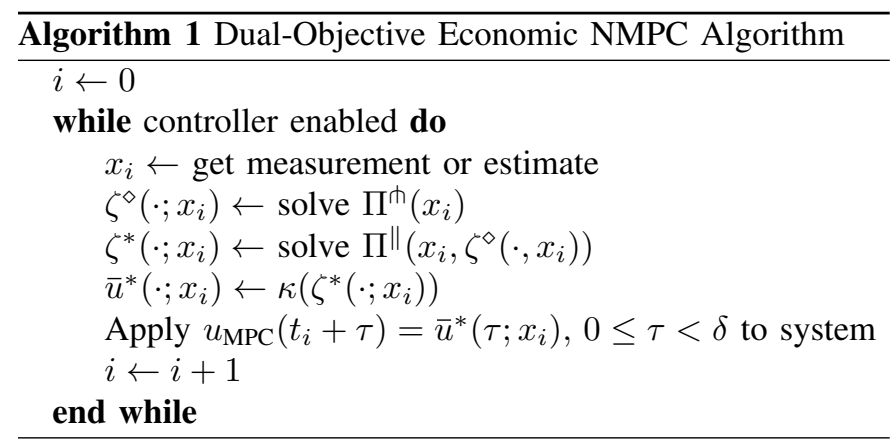

Remark 2. A common method to deal with infeasible state constraints is to soften them [20], constraint violation is then penalized using an exact penalty function. Such a method can in principle be used to enforce convergence to the set $\mathcal{N}$ also. Parallels can be drawn between such an approach and the technique presented in this paper, considering $R \rightarrow 0$, resulting in a cheap-control problem. Further discussion is however, beyond the scope of this paper.

\section{Properties of the Control Scheme}

The theoretical properties of the proposed dual-objective NMPC scheme are discussed below. First consider the definition of an admissible set, and a maximum admissible set:

Definition 1 (Admissible state set $\mathcal{X}_{0}$ [18]). A set $\mathcal{X}_{0} \subseteq \mathbb{R}^{n}$ is called admissible for $\Pi^{\dagger}\left(x_{0}\right)$ (6) if for all $x_{0} \in \mathcal{X}_{0}$ there exist admissible inputs $\tilde{v} \in \mathcal{L}^{\infty}\left(\mathbb{R}_{0}^{+}, \mathbb{R}^{l}\right), \tilde{w} \in \mathcal{L}^{\infty}\left(\mathbb{R}_{0}^{+}, \mathbb{R}^{m-l}\right)$, such that

$$
\begin{aligned}
\text { 1) } & \kappa\left(\xi\left(\tau ; x_{0}, \tilde{v}\right), \eta\left(\tau ; x_{0}, \tilde{v}, \tilde{w}\right), \tilde{v}(\tau), \tilde{w}(\tau)\right) \in \mathcal{U} \\
\text { 2) } & \xi\left(T^{\pitchfork} ; x_{0}, \tilde{v}\right) \in \mathcal{E}_{\xi}, \\
\text { 3) } & \eta\left(T^{\pitchfork} ; x_{0}, \tilde{v}, \tilde{w}\right) \in \mathcal{E}_{\eta} .
\end{aligned}
$$

Definition 2 (Maximum admissible set $\mathcal{R}_{0}[18]$ ). The maximum admissible set $\mathcal{R}_{0}$ is such that $\Pi^{\dagger}\left(x_{0}\right)$ (6) admits, for all $x_{0} \in \mathcal{R}_{0}$, an optimal (not necessarily unique) solution.
Proposition 1 (Recursive feasibility). Consider the application of Algorithm 1 to system (1). Let Assumptions 2 and 6 hold. Then, for all $x_{0} \in \mathcal{R}_{0}, \Pi^{\dagger}$ (6) and $\Pi^{\|}$(11) are feasible for all $t_{i} \geq t_{0}$.

Proof. The proof is structured as follows, it first shows that from the solution to $\Pi^{\dagger}\left(x_{i}\right)$ an admissible solution candidate to $\Pi^{\|}\left(x_{i}, \zeta^{\diamond}\left(\cdot, x_{i}\right)\right)$ can be derived. Subsequently, it is pointed out that $\Pi^{\|}$explicitly ensures the existence of a feasible solution to $\Pi^{\dagger}\left(x_{i+1}\right)$ through constraint $(11 \mathrm{~g})$, which yields recursive feasibility.

Consider any recalculation instant $t_{i}$, for which a solution to $\Pi^{\dagger}\left(x_{i}\right)$ exists (e.g., $\left.t_{0}\right)$. Now assume, w.l.o.g., that $T^{\pitchfork}=T^{\|}$and choose $(\bar{\xi}, \bar{\eta}, \bar{v}, \bar{w})=\zeta^{\diamond}\left(\cdot ; x_{i}\right)$. For unequal time horizons either truncate $\zeta^{\diamond}\left(\cdot ; x_{i}\right)$ or extend it via Assumption 6 . This solution candidate readily satisfies constraints (11b)-(11e). Feasibility of (11f) follows from $E\left(\tilde{\xi}^{\diamond}(\tau)\right) \leq$ $\max \left\{\varepsilon_{\mathcal{N}}^{2}, E\left(\tilde{\xi}^{\diamond}(\tau)\right)\right\} \forall \tau \in\left[0, T^{\|}\right]$and feasibility of $\tilde{\xi}^{\diamond}(\cdot)$ in $(11 \mathrm{~b})-(11 \mathrm{e})$. It remains to be shown that $(11 \mathrm{~g})$ is also satisfied. Through the terminal constraints in (6f), it holds that $\bar{\xi}^{\diamond}\left(T^{\pitchfork} ; x_{i}\right) \in \mathcal{E}_{\xi}$ and $\bar{\eta}^{\diamond}\left(T^{\pitchfork} ; x_{i}\right) \in \mathcal{E}_{\eta}$, and therefore by Assumption 6 there exist admissible inputs $\hat{v}_{\mathcal{E}}, \hat{w}_{\mathcal{E}}$ for a time interval of length $\delta$ that render the terminal sets forward invariant. Feasible trajectories with respect to (10a) and (10c) therefore exist. Picking any such input trajectory allows defining:

$$
\begin{gathered}
\tilde{v}\left(\tau-\delta ; \bar{x}_{i+1}^{\diamond}\right)=\left\{\begin{array}{l}
\bar{v}^{\diamond}\left(\tau ; x_{i}\right), \tau \in\left[\delta, T^{\pitchfork}\right] \\
\hat{v}_{\mathcal{E}}\left(\tau-T^{\pitchfork}\right), \tau \in\left(T^{\pitchfork}, T^{\pitchfork}+\delta\right]
\end{array},\right. \\
\tilde{w}\left(\tau-\delta ; \bar{x}_{i+1}^{\diamond}\right)=\left\{\begin{array}{l}
\bar{w}^{\diamond}\left(\tau ; x_{i}\right), \tau \in\left[\delta, T^{\dagger}\right] \\
\hat{w}_{\mathcal{E}}\left(\tau-T^{\dagger}\right), \tau \in\left(T^{\pitchfork}, T^{\pitchfork}+\delta\right]
\end{array}\right.
\end{gathered}
$$

The optimal value function $V^{\pitchfork}\left(x_{i}\right)=J^{\pitchfork}\left(\bar{\xi}^{\diamond}\left(\cdot ; x_{i}\right), \bar{v}^{\diamond}\left(\cdot ; x_{i}\right)\right)$ resulting from solving $\Pi^{\dagger}\left(x_{i}\right)$ and the cost $J^{\pitchfork}\left(\xi\left(\cdot ; \bar{x}_{i+1}^{\diamond}, \tilde{v}\left(\cdot ; \bar{x}_{i+1}^{\diamond}\right)\right), \tilde{v}\left(\cdot ; \bar{x}_{i+1}^{\diamond}\right)\right) \quad$ evaluated for the admissible inputs (13). Subtracting the two yields

$$
\begin{aligned}
& J^{\dagger}\left(\xi\left(\cdot ; \bar{x}_{i+1}^{\diamond}, \tilde{v}\left(\cdot ; \bar{x}_{i+1}^{\diamond}\right)\right), \tilde{v}\left(\cdot ; \bar{x}_{i+1}^{\diamond}\right)\right)-V^{\pitchfork}\left(x_{i}\right)= \\
& -\int_{0}^{\delta} F^{\dagger}\left(\bar{\xi}^{\diamond}\left(\tau ; x_{i}\right), \bar{v}^{\diamond}\left(\tau ; x_{i}\right)\right) \mathrm{d} \tau \\
& +\int_{T^{\dagger}-\delta}^{T^{\dagger}} F^{\pitchfork}\left(\xi\left(\tau ; \bar{x}_{i+1}^{\diamond}, \tilde{v}\left(\cdot ; \bar{x}_{i+1}^{\diamond}\right)\right), \tilde{v}\left(\tau ; \bar{x}_{i+1}^{\diamond}\right)\right) \mathrm{d} \tau \\
& -E\left(\bar{\xi}^{\diamond}\left(T^{\pitchfork} ; x_{i}\right)\right)+E\left(\xi\left(T^{\pitchfork} ; \bar{x}_{i+1}^{\diamond}, \tilde{v}\left(\cdot ; \bar{x}_{i+1}^{\diamond}\right)\right)\right) .
\end{aligned}
$$

Due to (13) satisfying (7c), zero is obtained as an upper bound to the last three terms and therefore it follows

$$
J^{\pitchfork}\left(\xi\left(\cdot ; \bar{x}_{i+1}^{\diamond}, \tilde{v}\left(\cdot ; \bar{x}_{i+1}^{\diamond}\right)\right), \tilde{v}\left(\cdot ; \bar{x}_{i+1}^{\diamond}\right)\right) \leq \tilde{J}^{\pitchfork}\left(x_{i}\right) .
$$

This shows that feasibility of $\Pi^{\dagger}$ implies feasibility of $(11 \mathrm{~g})$ in $\Pi^{\|}$. No disturbances and plant-model mismatch are considered and therefore the state evolves as predicted in the interval $t \in\left[t_{i}, t_{i+1}\right]$, hence $\xi\left(t_{i+1}\right)=\bar{\xi}^{*}\left(\delta ; x_{i}\right), \eta\left(t_{i+1}\right)=\bar{\eta}^{*}\left(\delta ; x_{i}\right)$. Through constraint $(11 \mathrm{~g})$ there exists a feasible solution at sampling time instant $t_{i+1}$ in $\Pi^{\pitchfork}$.

Proposition 2 (Convergence of $\xi(\cdot)$ to $\mathcal{N}$ ). Consider the application of Algorithm 1 to system (1) and suppose that Assumptions 2, 5-6 hold. Then, for all $x_{0} \in \mathcal{R}_{0}$, it holds (in TNF coordinates) that $\left.\lim _{t \rightarrow \infty} \| \xi\left(t ; x_{0}\right)\right) \|_{\mathcal{N}}=0$. 
Proof. The proof consists of two steps. First it is shown that the state $\xi$ converges to the target neighborhood $\mathcal{N}$ of the manifold through $(11 \mathrm{~g})$ enforcing a monotonic decrease of a Lyapunov-like value function. Thereafter it is shown that the same condition ensures forward invariance of the set $\mathcal{N}$ for an appropriate choice of $\bar{j}^{\dagger}$ for (10b).

Step 1: First the case is considered where at the current time instant $t_{i}$ the optimal value function associated to $\Pi^{\dagger}$ satisfies $V^{\pitchfork}\left(x_{i}\right)>\varepsilon_{\mathcal{N}}^{2}$. Due to optimality $V^{\pitchfork}\left(x_{i+1}\right)$ is bounded from above by $J^{\pitchfork}\left(\xi^{\diamond}\left(\cdot ; x_{i+1}\right), \tilde{v}\left(\cdot ; x_{i+1}\right)\right)$; i.e. $V^{\pitchfork}\left(x_{i+1}\right)-$ $V^{\pitchfork}\left(x_{i}\right) \leq J^{\pitchfork}\left(\xi^{\diamond}\left(\cdot ; x_{i+1}\right)-V^{\pitchfork}\left(x_{i}\right)\right.$. Lower boundedness of $F^{\pitchfork}$ by $\gamma \in \mathcal{K}$ and $(7 \mathrm{c})$ yield

$$
\begin{aligned}
V^{\pitchfork}\left(x_{i+1}\right)-V^{\pitchfork}\left(x_{i}\right) \leq-\int_{0}^{\delta} & \gamma\left(\left\|\bar{\xi}^{\diamond}\left(\tau ; x_{i}\right)\right\|\right) \mathrm{d} \tau \leq \\
& -\int_{0}^{\delta} \gamma\left(\left\|\bar{\xi}^{\diamond}\left(\tau ; x_{i}\right)\right\|_{\mathcal{N}}\right) \mathrm{d} \tau
\end{aligned}
$$

whereby the last inequality follows from the definition of $\mathcal{N}$ in (9). No plant-model mismatch and disturbances are considered, therefore the predicted trajectory $\bar{\xi}\left(\tau ; x_{i}, \bar{v}^{\diamond}\left(\cdot ; x_{i}\right)\right)$ and the true state evolution $\xi\left(\tau ; x_{i}, \bar{v}^{\diamond}\left(\cdot ; x_{i}\right)\right)$ coincide for $\tau \in\left[t_{i}, t_{i+1}\right]$. Hence, $V^{\pitchfork}\left(x_{i}\right)>\varepsilon_{\mathcal{N}}^{2}$ strictly decreases between $t_{i}$ and $t_{i+1}$. Thus, $\xi$ will eventually enter the set $\mathcal{N}$.

Step 2: Now consider that at time $t_{i}$ it is the case that $V^{\pitchfork}\left(x_{i}\right) \leq \varepsilon_{\mathcal{N}}^{2}$. Recall that $\left(\xi_{i}, \eta_{i}\right)=\Phi\left(x_{i}\right)$. As a consequence of the neighborhood $\mathcal{N}$ being a level set of the value function $\xi^{\top} P \xi$ of the infinite-horizon LQR problem (4a) and (5), it must hold that $\xi_{i} \in \mathcal{N}$. Moreover, there exists a trajectory starting from $\xi_{i}$ which remains in $\mathcal{N}$ for all $t \geq t_{i}$. Now, consider the following observations:

- The cost functional (5a) is lower bounded by the optimal LQR value function.

- The value of the optimal LQR cost on $\partial \mathcal{N}$ is $\varepsilon_{\mathcal{N}}^{2}$.

Due to Assumption 2, any trajectory starting inside the set $\mathcal{N}$ that makes an excursion outside $\mathcal{N}$ crosses $\partial \mathcal{N}$ at least once. Hence any such trajectory violates constraint (11g). Finally, due to the already established recursive feasibility, considering no plant-model mismatch and disturbances, the optimal cost of $\Pi^{\dagger}$ at the next sampling time instance is upper bounded by $\varepsilon_{\mathcal{N}}^{2}$; which concludes the proof.

Theorem 1. Let Assumptions 1-6 hold. Then, for all $x_{0} \in \mathcal{R}_{0}$, system (1) controlled by Algorithm 1 solves Problem 1.

Proof. First it is shown that Proposition 2 is sufficient to state Problem 1a is solved by Algorithm 1. Subsequently, it is shown that $\Pi^{\|}$minimizes $J^{\text {eco }}$, and that increasing $\varepsilon$ grows the feasible set of $\Pi^{\|}$and therefore enables improving the economic performance as stated in Problem 1b. The proof concludes with showing Problem 1c is solved.

(a) Consider the vector $\xi_{1}$ corresponding to $\alpha_{1}, \ldots, \alpha_{l}$ and $\xi_{2}$ the vector of remaining elements corresponding to the first and higher time derivatives of $\alpha_{1}, \ldots, \alpha_{l}$. In Proposition 2 it is established that $\lim _{t \rightarrow \infty}\|\xi(t)\|_{P} \leq \varepsilon_{\mathcal{N}}$. To relate this result to statement $(a)$ consider the set $\mathcal{N}_{1}=\left\{\xi_{1} \in \mathbb{R}^{l}\right.$ $\left.\exists \xi_{2}:\|\xi(t)\|_{P} \leq \varepsilon_{\mathcal{N}}\right\}$. A candidate for $\varepsilon$ is $\varepsilon=\min \{r \in$ $\left.\mathbb{R} \mid \mathcal{N}_{1} \subseteq \mathcal{B}(r)\right\}$, with $\mathcal{B}(r)=\left\{\xi_{1} \in \mathbb{R}^{l} \mid\left\|\xi_{1}\right\|_{2} \leq r\right\}$. Observe that since the set $\mathcal{N}_{1}$ is compact, $\varepsilon \in(0, \infty)$ exists.
Assumptions 3 and 4 are sufficient for (1) to be equivalent to (4) under the transversely linearizing feedback (3), see [3, Thm. 3.2]. Convergence of $\xi$ to a neighborhood $\mathcal{N}$ of the origin implies convergence to an $\varepsilon$-distance of the manifold $\mathcal{M}^{\star}$ in the state-space of (1).

(b) Recall that $\Phi$ is a diffeomorphism, $\kappa$ is regular. Let $\mathcal{D}_{1}=\mathbb{R}^{n} \times \mathcal{U}$ and $\mathcal{D}_{2}=\{(\xi, \eta, v, w) \mid \kappa(\xi, \eta, v, w) \in \mathcal{U}\}$. By construction, $F^{\mathrm{eco}}\left(\mathcal{D}_{1}\right)=F^{\|}\left(\mathcal{D}_{2}\right)$. Hence, Algorithm 1 optimizes the user-specified economic cost through minimizing $J^{\|}$. Next, consider the parameterized set $\mathcal{N}\left(\varepsilon_{\mathcal{N}}\right)=\left\{\xi \in \mathbb{R}^{n} \mid\right.$ $\left.\xi^{\top} P \xi \leq \varepsilon_{\mathcal{N}}^{2}\right\}$. Let $V^{\|}\left(x_{i}, \varepsilon_{\mathcal{N}}\right)$ be the optimal value function of $\Pi^{\|}\left(x_{i}\right)$ for a specific value of $\varepsilon_{\mathcal{N}}$. Observe that increasing $\varepsilon_{\mathcal{N}}$ relaxes the constraints of $\Pi^{\|}$, i.e. $\mathcal{N}\left(\varepsilon_{1}\right) \supset \mathcal{N}\left(\varepsilon_{2}\right)$. Therefore it immediately follows that $V^{\|}\left(x_{i}, \varepsilon_{1}\right) \leq V^{\|}\left(x_{i}, \varepsilon_{2}\right)$.

(c) The NMPC scheme described in Algorithm 1 explicitly handles the input constraints $u \in \mathcal{U}$ using the input transformation $\kappa$. In combination with the recursive feasibility result of Proposition 1 this shows item (c) and concludes the proof.

\section{REMARKS ON NUMERICAL IMPLEMENTATION}

The optimal control problems $\Pi^{\pitchfork}$ and $\Pi^{\|}$, defined in (6) and (11), respectively, are formulated in the TNF coordinates $(\xi, \eta)$. However, feedback linearization strategies, and therefore also the TNF coordinate description, suffer from a few notable drawbacks:

- Whereas the original state-space coordinates usually correspond to physical quantities, the TNF coordinates are mostly abstract quantities with no obvious physical meaning. The same applies to the control input $u$ and the TNF inputs $v$ and $w$. Constraints on $x$ and $u$ become highly nonlinear in $\xi, \eta, v$ and $w$ due to the nonlinearity of the inverse state transformation $\Phi^{-1}: \mathbb{R}^{n-n^{\star}} \times$ $\mathbb{R}^{n^{\star}} \rightarrow \mathbb{R}^{n}$ and the transversely linearizing feedback $\kappa: \mathbb{R}^{l} \times \mathbb{R}^{m-l} \rightarrow \mathbb{R}^{m}$.

- The diffeomorphism $\Phi$ and the transversely linearizing feedback $\kappa$ are typically locally defined and often suffer from numerically undesirable properties such as singularities.

In the traditional context of (transverse) feedback linearization (e.g., [4]), facilitating linear control design techniques, advantages may considerably outweigh these disadvantages. However, NMPC naturally handles nonlinear systems, bringing the usefulness of implementing the OCPs in TNF coordinates into question. Inspired by [5], the suggested modus operandi is therefore to employ the transverse normal form description as a tool during the design of a manifold stabilizing controller, but to implement OCPs in terms of $\bar{x}$ and $\bar{u}$. In contrast to the approaches presented in $[1,6]$, a realization of the manifold convergence condition $(11 \mathrm{~g})$ in $\Pi^{\|}$can be formulated in $(x, u)$ coordinates. For example, consider the approach where the $\hat{v}$ and $\hat{w}$ trajectories for which constraints (10a)-(10c) must hold are explicitly computed. The resulting transverse OCP then reads as:

$$
\begin{aligned}
\min _{\bar{x}, \bar{u}} & J^{\pitchfork}(\bar{x}, \bar{u}) \\
\text { s.t. } & \dot{\bar{x}}=f(\bar{x})+G(\bar{x}) \bar{u} \\
& \bar{x}(0)=x_{i}, \bar{u} \in \mathcal{U}, \bar{x}\left(T^{\pitchfork}\right) \in \mathcal{E}_{x},
\end{aligned}
$$


with $\mathcal{E}_{x} \subseteq \Phi^{-1}\left(\mathcal{E}_{\xi} \times \mathcal{E}_{\eta}\right)$, and

$$
\begin{aligned}
J^{\pitchfork}(\hat{x}, \hat{u})=\int_{0}^{T^{\dagger}}\|\boldsymbol{\xi}(\hat{x}(\tau))\|_{Q}^{2}+ & \|\boldsymbol{v}(\hat{x}(\tau), \hat{u}(\tau))\|_{R}^{2} \mathrm{~d} \tau \\
& +\left\|\boldsymbol{\xi}\left(\hat{x}\left(T^{\pitchfork}\right)\right)\right\|_{P}^{2},
\end{aligned}
$$

where the mappings $\boldsymbol{\xi}: \mathbb{R}^{n} \rightarrow \mathbb{R}^{n-n^{\star}}$ and $\boldsymbol{v}: \mathbb{R}^{n} \times \mathbb{R}^{m} \rightarrow$ $\mathbb{R}^{l}$ are composed of $\alpha_{1}, \ldots, \alpha_{l}$ and a finite number of their derivatives, see Section III-A. The second OCP reads as:

$$
\begin{aligned}
\min _{\bar{x}, \bar{u}, \hat{x}, \hat{u}} & J^{\|}(\bar{x}, \bar{u}) \\
\text { s.t. } & \dot{\bar{x}}=f(\bar{x})+G(\bar{x}) \bar{u} \\
& \bar{x}(0)=x_{i}, \bar{u} \in \mathcal{U} \\
& E\left(\boldsymbol{\xi}(\bar{x}(\tau)) \leq \max \left\{\varepsilon_{\mathcal{N}}^{2}, E(\tilde{\xi}(\tau))\right\} \forall \tau \in\left[0, T^{\|}\right]\right. \\
& \dot{\hat{x}}=f(\hat{x})+G(\hat{x}) \hat{u} \\
& \hat{x}(\delta)=\bar{x}(\delta), \hat{u} \in \mathcal{U}, \hat{x}\left(\delta+T^{\pitchfork}\right) \in \mathcal{E}_{x} \\
& J^{\dagger}(\hat{x}, \hat{u}) \leq \tilde{J}^{\dagger}\left(x_{i}\right) .
\end{aligned}
$$

Observe that the support of $\bar{x}$ and $\bar{u}$, on the one hand, and $\hat{x}$ and $\hat{u}$, on the other hand, in the definition for $\Pi^{\|}$above are $\left[0, T^{\|}\right]$and $\left[\delta, \delta+T^{\dagger}\right]$, respectively.

The control horizons of $\Pi^{\dagger}$ and $\Pi^{\|}$can be chosen independently. This is a particularly useful property of the control scheme when considering real-time implementation aspects. By increasing the value for $T^{\pitchfork}$, the size of the maximum admissible set $\mathcal{R}_{0}$ can be increased, whereas increasing $T^{\|}$is in the benefit of closed-loop economic performance. Decreasing the length of any of the two time horizons will reduce the computational complexity of the overall control scheme, enabling the user to effectively trade off the size of the maximum admissible set, the economic optimality and the computational complexity. A typical implementation of Algorithm 1 uses a direct solution approach [21], approximating the infinitedimensional OCPs with Non-Linear Programs (NLPs). It is highlighted that the first OCP, $\Pi^{\dagger}$, is a constrained leastsquares type problem and can therefore be efficiently solved using the Generalized Gauss-Newton method. The second OCP, $\Pi^{\|}$, is more challenging to solve due to its general objective function and the increased problem dimension. Moreover, upon application one may resort to using approximate solution methods, potentially using the solution of $\Pi^{\dagger}$ as initial guess.

\section{TUTORIAL EXAMPLE}

The example shows the application of the control approach to a quadrotor flying in a plane. Considered is a onedimensional manifold in the output space of the quadrotor. The states $x=\left[\begin{array}{lllll}x_{p} & \dot{x}_{p} & z_{p} & \dot{z}_{p} & \theta\end{array}\right]^{\top}$ are governed by the inputs $u=\left[\begin{array}{ll}F_{t} & \omega\end{array}\right]^{\top}$ (thrust and angular velocity) and the set of differential equations:

$$
\frac{\mathrm{d} x}{\mathrm{~d} t}=f(x)+G(x) u=\left[\begin{array}{c}
\dot{x}_{p} \\
0 \\
\dot{z}_{p} \\
-g \\
0
\end{array}\right]+\left[\begin{array}{cc}
0 & 0 \\
-\sin (\theta) & 0 \\
0 & 0 \\
\cos (\theta) & 0 \\
0 & 1
\end{array}\right]\left[\begin{array}{c}
F_{t} \\
\omega
\end{array}\right]
$$

where $g=9.81 \mathrm{~m} / \mathrm{s}^{2}$ denotes the gravitational acceleration constant. The output map reads $y=h(x)=\left[\begin{array}{ll}x_{p} & z_{p}\end{array}\right]^{\top}$. The inputs are limited by $0 \leq F_{t} \leq 15 \mathrm{~N}$ and $|\omega| \leq 2 \pi \mathrm{rad} / \mathrm{s}$. The prioritized control objective is stabilizing the manifold defined by the implicit equation:

$$
\alpha(x)=\sigma(h(x))=x_{p}^{2}+z_{p}^{2}-r^{2}
$$

a circle in the output space with radius $r=1 \mathrm{~m}$. Both the system dynamics and the manifold are thus nonlinear. In order to find the vector relative degree, compute:

$$
\begin{aligned}
& L_{g_{1}} \alpha(x)=0, L_{g_{2}} \alpha(x)=0, L_{g_{2}} L_{f} \alpha(x)=0 \\
& L_{g_{1}} L_{f} \alpha(x)=-2 x_{p} \sin (\theta)+2 z_{p} \cos (\theta) .
\end{aligned}
$$

Hence, the system has a relative degree $\rho=2$ for the input $F_{t}$ to $\alpha(x)$. This is the basis of the TNF construction. Note that $L_{g_{1}} L_{f} \alpha(x)$ can become zero, particularly when the vertical axis of the quadrotor is aligned with the tangent vector of the manifold. This is most apparent when the tangent vector of the manifold is (nearly) parallel to the $z_{p}$-axis: in that case, when the quadrotor is oriented straight $(\theta=0)$, the input $F_{t}$ cannot stabilize the manifold. The result is a singularity in the transversely linearizing feedback and the dynamics described in the TNF coordinates. Therefore, the approach in Section V is followed to formulate OCPs (17) and (18); working with the pysical states $x$ instead of the TNF coordinates $(\xi, \eta)$. In addition, extra care is required in the construction of the terminal set $\mathcal{E}_{\eta}$.

\section{A. Transverse Normal Form Description}

A suitable diffeomorphism mapping between the original states and the transverse normal form is:

$$
\begin{gathered}
\Phi(x)=\left[\begin{array}{c}
\xi_{1} \\
\xi_{2} \\
\eta_{1} \\
\eta_{2} \\
\eta_{3}
\end{array}\right]=\left[\begin{array}{c}
x_{p}^{2}+z_{p}^{2}-r^{2} \\
2 x_{p} \dot{x}_{p}+2 z_{p} \dot{z}_{p} \\
\arg \left(z_{p}-x_{p} i\right) \\
\frac{x_{p} \dot{p}_{p}-z_{p} \dot{x}_{p}}{x_{p}^{2}+z_{p}^{2}} \\
\theta
\end{array}\right] \\
\Phi^{-1}(\xi, \eta)=\left[\begin{array}{c}
x_{p} \\
\dot{x}_{p} \\
z_{p} \\
\dot{z}_{p} \\
\theta
\end{array}\right]=\left[\begin{array}{c}
-\sin \left(\eta_{1}\right) \sqrt{\xi_{1}+r^{2}} \\
-\frac{\xi_{2} \sin \left(\eta_{1}\right)+2 \eta_{2} \cos \left(\eta_{1}\right)\left(\xi_{1}+r^{2}\right)}{2 \sqrt{\xi_{1}+r^{2}}} \\
\cos \left(\eta_{1}\right) \sqrt{\xi_{1}+r^{2}} \\
\frac{\xi_{2} \cos \left(\eta_{1}\right)-2 \eta_{2} \sin \left(\eta_{1}\right)\left(\xi_{1}+r^{2}\right)}{2 \sqrt{\xi_{1}+r^{2}}} \\
\eta_{3}
\end{array}\right] .
\end{gathered}
$$

The according transversely linearizing feedback reads:

$$
u=\tilde{\kappa}(x, v, w)=\left[\begin{array}{c}
\frac{v-2 \dot{x}_{p}^{2}+2 g z_{p}-2 \dot{z}_{p}^{2}}{2 z_{p} \cos (\theta)-2 x_{p} \sin (\theta)} \\
w
\end{array}\right],
$$

and $\kappa(\xi, \eta, v, w)=\tilde{\kappa}\left(\Phi^{-1}(\xi, \eta), v, w\right)$. For this example the transverse running cost uses

$$
Q=\left[\begin{array}{ll}
1 & 0 \\
0 & 1
\end{array}\right], R=0.001
$$

The terminal cost weighting matrix $P$ is chosen according to the solution of $(5 \mathrm{c})$. The LQR transverse feedback law, feasible in the terminal set $\mathcal{E}$ is defined as $v_{\mathrm{LQR}}(\xi)=-K \xi$, with $K=R^{-1} B^{\top} P$. The tangential running cost maximizes the velocity $\eta_{2}$, by which $\eta_{1}$ is maximized in each open-loop prediction:

$$
F^{\|}(x, u)=\frac{z_{p} \dot{x}_{p}-x_{p} \dot{z}_{p}}{x_{p}^{2}+z_{p}^{2}} .
$$




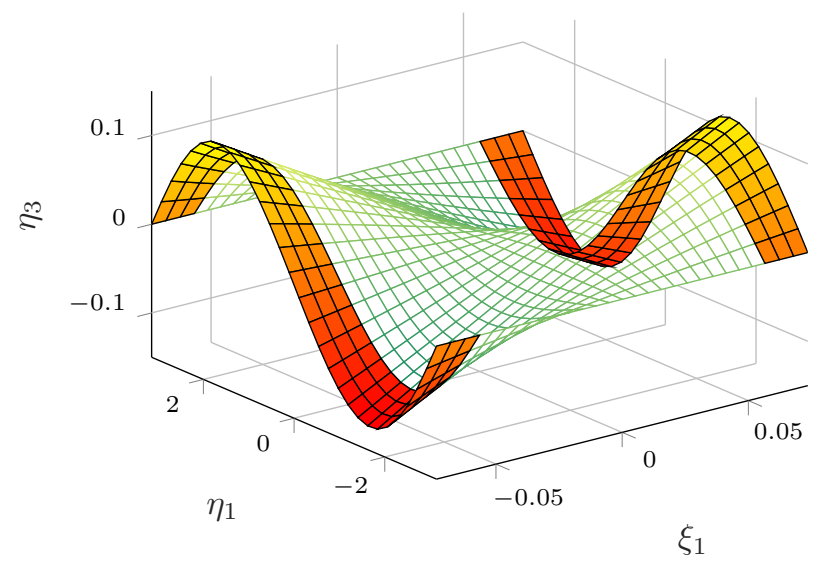

Fig. 1. Projection of the terminal set $\mathcal{E}_{\eta}(\xi)$ for $\left(\xi_{2}, \eta_{2}\right)=(0,0)$. Along the $\xi_{1}$-axis the terminal set is bounded through $\varrho^{*}$.

\section{B. Computing the Terminal Set}

First, recall that under the transverse feedback $v_{\mathcal{E}}:=$ $v_{\mathrm{LQR}}(\xi)$ a set $\mathcal{E}_{\xi}(\varrho)=\left\{\xi \in \mathbb{R}^{2} \mid \xi^{\top} P \xi \leq \varrho\right\}$ is rendered forward invariant. As preferably a large terminal set is obtained, the parameter $\varrho$ is maximized. To reduce the complexity of the example, no terminal feedback law is determined for the tangential dynamics. Instead, the tangential velocity and acceleration are constrained to zero:

$$
\mathcal{E}_{\eta}(\xi)=\left\{\eta \in \mathbb{R}^{3} \mid \eta_{2}=0, f_{2}^{0}(\xi, \eta)=G_{2,1}^{\pitchfork}(\xi, \eta) v_{\mathrm{LQR}}(\xi)\right\},
$$

where $f_{2}^{0}$ refers to the second element of the vector-valued function $f^{0}$. Likewise, $G_{2,1}^{\pitchfork}$ selects the second row and first column of the matrix valued function $G^{\pitchfork}$. Figure 1 visualizes one projection of the terminal set. It shows the required orientation $\eta_{3}=\theta$ of the quadrotor for different positions on and transverse to the manifold. The parameter $\varrho$ can be maximized by solving the following optimization problem:

$$
\begin{aligned}
\max _{\varrho, \xi, \eta} \varrho & \\
\text { s.t. } & \forall \xi \in \partial \mathcal{E}_{\xi}(\varrho): \forall \eta \in \mathcal{E}_{\eta}(\xi): \\
& \kappa\left(\xi, \eta, v_{\mathrm{LQR}}, 0\right) \in \mathcal{U} .
\end{aligned}
$$

The above semi-infinite program is approximated with a nonlinear program. A finite set of samples are taken from $\partial \mathcal{E}_{\xi}(\varrho)$ and for every possible combination with a finite number of samples from $\left\{\eta_{1} \in \mathbb{R} \mid-\pi \leq \eta_{1} \leq \pi\right\}$, inputfeasibility constraints are introduced. The obtained solution is $\varrho^{*}=0.0033$.

\section{Computing the Target Neighborhood}

The target neighborhood $\mathcal{N}$ is derived from the target tolerance $\varepsilon$ specified by the practitioner in Problem 1a. The value for $\varepsilon$ is defined as a bound on $\xi_{1}$, whereas the target neighborhood is defined as $E(\xi)=\xi^{\top} P \xi \leq \varepsilon_{\mathcal{N}}^{2}$. The aim is to find the least conservative choice of $\varepsilon_{\mathcal{N}}$, such that Problem 1a is always satisfied. It follows that $\varepsilon_{\mathcal{N}}$ can be found through the following relation:

$$
\varepsilon_{\mathcal{N}}=\frac{\varepsilon}{\sqrt{P_{1,1}^{-1}}},
$$

TABLE I

THE FOUR INITIAL STATES CONSIDERED IN THE SIMULATIONS.

$$
\begin{array}{lllll}
x_{0,1}: & {\left[\begin{array}{lllll}
0 & 0 & 1.5 & 0 & 0
\end{array}\right]^{\top}} \\
x_{0,2}: & {\left[\begin{array}{lllll}
0 & 0 & 0.5 & 3 & 0
\end{array}\right]^{\top}} \\
x_{0,3}: & {\left[\begin{array}{lllll}
0 & 0 & 0.1 & 0 & 0
\end{array}\right]^{\top}} \\
x_{0,4}: & {\left[\begin{array}{lllll}
0 & 1 & 1.5 & 0 & 0
\end{array}\right]^{\top}}
\end{array}
$$

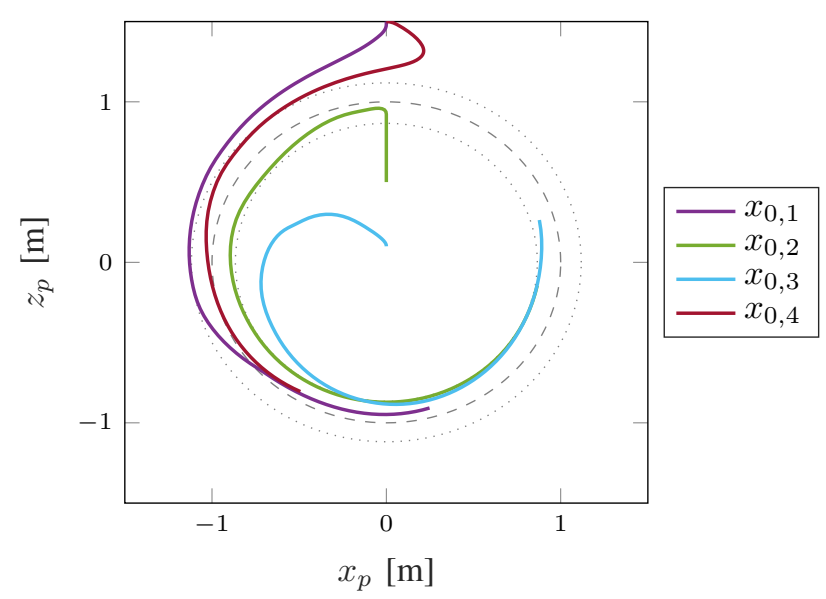

Fig. 2. Simulated trajectories (2s) for the initial states in Table I, with $\varepsilon=$ 0.25 . The dashed line depicts the manifold, the dotted lines depict the bounds imposed via the target tolerance.

where $P_{1,1}^{-1}$ denotes the upper-left entry of the matrix $P^{-1}$.

\section{Simulation Results}

The prediction horizons are set to $T^{\pitchfork}=1 \mathrm{~s}$ and $T^{\|}=1.2 \mathrm{~s}$. A direct multiple shooting approach is used to approximate the optimal control problems (17) and (18) with NLPs. A uniform discretization step size of $\Delta t=0.02 s$ is selected. CasADi [22] is used in conjunction IPOPT [23] to formulate and solve the NLPs for simulations in MATLAB.
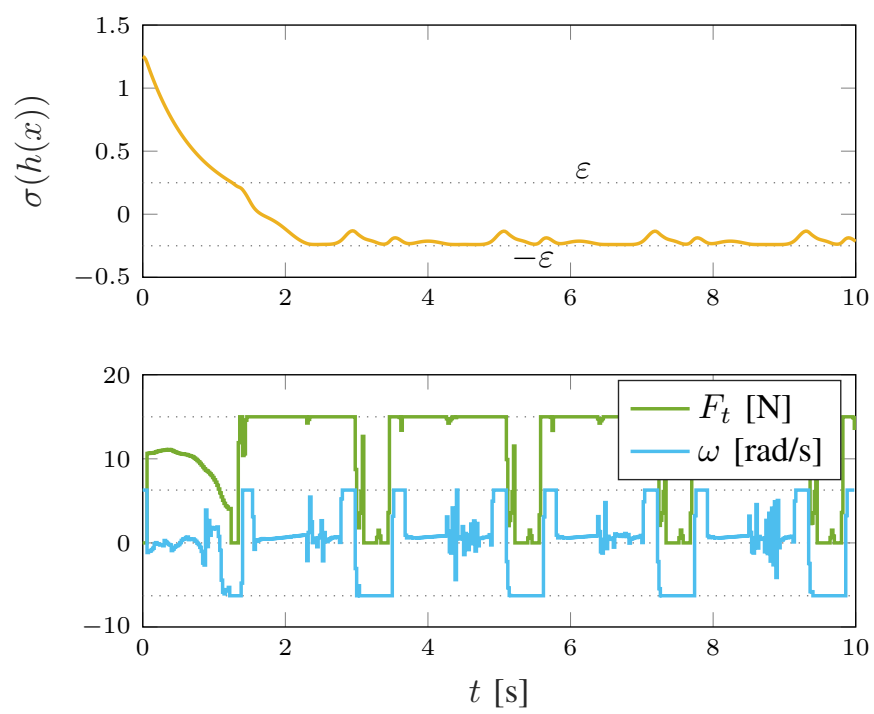

Fig. 3. Evolution of output function and the control trajectory for initial state $x_{0,4}$, see Table I. 
TABLE II

AVERAGE NUMBER OF SECONDS PER REVOLUTION FOR DIFFERENT VALUES OF $\varepsilon$ IN A 10 SECOND SIMULATION. INITIAL STATE: $x_{0}=\left[\begin{array}{lllll}0 & -0.1 & 1 & 0 & 0\end{array}\right]^{\top}$. THE LAST COLUMN DEPICTS THE SHORTEST TIME FOR ONE REVOLUTION CONSIDERING A PERIODIC BOUNDARY CONDITION (PBC) FOR THE BEGIN AND END STATE.

\begin{tabular}{cccc} 
& \multicolumn{2}{c}{ Algorithm 1 } & \\
\cline { 2 - 3 }$\varepsilon$ & $T^{\|}=0.4 s$ & $T^{\|}=1.2 s$ & PBC \\
\hline 0.005 & 2.4210 & 2.4248 & 2.2862 \\
0.01 & 2.3480 & 2.3278 & 2.2758 \\
0.05 & 2.3092 & 2.2852 & 2.2282 \\
0.1 & 2.2940 & 2.2516 & 2.1925 \\
0.25 & 2.2834 & 2.1769 & 2.0894 \\
0.7 & 2.1753 & 1.9547 & 1.6827
\end{tabular}

Figure 2 depicts trajectories for the four different initial states listed in Table I, notice how the controller stabilizes the manifold $\mathcal{M}$ and simultaneously moves in a counterclockwise direction along the manifold. Figure 3 shows the evaluation of the function $\alpha$ and the input trajectories in time for $x_{0,4}$ in Figure 2. As is expected with an objective that aims to maximize speed, the actuation is limited by its constraints most of the time. Only a slight degree of conservatism can be observed in Figure 3, originating from the selection of $\varepsilon_{\mathcal{N}}$ from $\varepsilon$. Also, it can be observed that the input $\omega$ exhibits jittering behavior at some points in time, explained by the lack of an input(-rate) penalty in the economic objective. Finally, the data in Table II illustrates that the economic objective improves for increasing values of $\varepsilon$, as mostly with increasing values for $T^{\|}$.

\section{CONCLUSION}

The paper presented a sequential dual-objective NMPC scheme with economic costs near manifolds. Given a transverse feedback linearizable system, a state-space manifold, an economic running cost describing the desired motion near the manifold, and a user-specified target neighborhood of the manifold, two optimal control problems are solved in sequence. The first OCP disregards the desired motion near the manifold, its solution serves to compute convergence conditions for the user-defined target neighborhood of the manifold. These convergence conditions are imposed in the second OCP, of which the solution optimizes economic tangential performance. The presented solution method relies on a transverse normal form description of the system dynamics, however, it is shown that a numerical implementation can take place in the original statespace coordinates. A tutorial example is presented for a planar quadrotor system which exposes a high correlation between the size of the target neighborhood and the achieved closedloop economic performance.

\section{ACKNOWLEDGMENT}

This work is supported by the EU via ITN-TEMPO (607 957), by the Research Foundation Flanders (FWO) via projects $\mathrm{G} 0 \mathrm{C} 4515 \mathrm{~N}$ and $\mathrm{G} 0 \mathrm{~A} 6917 \mathrm{~N}$, and benefits from support from KU Leuven-BOF PFV/10/002 Centre of Excellence: Optimization in Engineering (OPTEC). Also, the support from Flanders Make-the Flemish strategic research centre for the manufacturing industry-is acknowledged.
Timm Faulwasser acknowledges funding from the BadenWürttemberg Stiftung under the Elite Programme for Postdocs.

\section{REFERENCES}

[1] N. van Duijkeren, T. Faulwasser, and G. Pipeleers, "NMPC with economic objectives on target manifolds," in Proceedings of the 56th IEEE Conference on Decision and Control (CDC), Melbourne, Australia, Dec. 2017.

[2] A. Banaszuk and J. Hauser, "Feedback linearization of transverse dynamics for periodic orbits," Systems \& Control Letters, vol. 26, no. 2, pp. $95-105,1995$.

[3] C. Nielsen and M. Maggiore, "On local transverse feedback linearization," SIAM Journal on Control and Optimization, vol. 47, no. 5, pp. 2227-2250, 2008.

[4] C. Nielsen, C. Fulford, and M. Maggiore, "Path following using transverse feedback linearization: Application to a maglev positioning system," Automatica, vol. 46, no. 3, pp. 585-590, 2010.

[5] T. Faulwasser and R. Findeisen, "Nonlinear model predictive control for constrained output path following," IEEE Transactions on Automatic Control, vol. 61, no. 4, pp. 1026-1039, 2016.

[6] M. Böck and A. Kugi, "Constrained model predictive manifold stabilization based on transverse normal forms," Automatica, vol. 74, pp. 315-326, 2016.

[7] M. Ellis, J. Liu, and P. Christofides, Economic Model Predictive Control. Springer-Verlag GmbH, 2016.

[8] T. Faulwasser, L. Grüne, and M. Müller, "Economic nonlinear model predictive control: Stability, optimality and performance," Foundations and Trends in Systems and Control, vol. 5, no. 1, pp. 1-98, 2018.

[9] M. Diehl, R. Amrit, and J. B. Rawlings, "A Lyapunov function for economic optimizing model predictive control," IEEE Transactions on Automatic Control, vol. 56, no. 3, pp. 703-707, 2011.

[10] D. Angeli, R. Amrit, and J. B. Rawlings, "On average performance and stability of economic model predictive control," IEEE Transactions on Automatic Control, vol. 57, no. 7, pp. 1615-1626, 2012.

[11] L. Grüne, "Economic receding horizon control without terminal constraints," Automatica, vol. 49, no. 3, pp. 725-734, 2013.

[12] T. Faulwasser and D. Bonvin, "On the design of economic NMPC based on approximate turnpike properties," in Proceedings of the 54th IEEE Conference on Decision and Control (CDC), Osaka, Japan, Dec. 2015.

[13] C. Böhm, R. Findeisen, and F. Allgöwer, "Avoidance of poorly observable trajectories: A predictive control perspective," in Proceedings of the 17th IFAC World Congress, Seoul, Korea, Jul. 2008.

[14] J. P. Maree and L. Imsland, "Combined economic and regulatory predictive control," Automatica, vol. 69, pp. 342-347, 2016.

[15] L. Grüne and J. Pannek, Nonlinear Model Predictive Control. Springer London, 2011.

[16] A. Isidori, Non-linear control systems. Springer London, 1995.

[17] H. Nijmeijer and A. van der Schaft, Nonlinear Dynamical Control Systems. Springer New York, 1990.

[18] R. Findeisen, "Nonlinear model predictive control: a sampled-data feedback perspective," Ph.D. dissertation, Universität Stuttgart, 2004.

[19] F. A. Fontes, "A general framework to design stabilizing nonlinear model predictive controllers," Systems \& Control Letters, vol. 42, no. 2, pp. 127-143, 2001.

[20] E. C. Kerrigan and J. M. Maciejowski, "Soft constraints and exact penalty functions in model predictive control," in Proceedings of the UKACC International Conference on Control, Cambridge, UK, Sep. 2000.

[21] H. G. Bock and K. J. Plitt, "A multiple shooting algorithm for direct solution of optimal control problems," in Proceedings of the 9th IFAC World Congress, Budapest, Hungary, Jul. 1984.

[22] J. A. E. Andersson, J. Gillis, G. Horn, J. B. Rawlings, and M. Diehl, "CasADi-a software framework for nonlinear optimization and optimal control," Mathematical Programming Computation, 2018.

[23] A. Wächter and L. T. Biegler, "On the implementation of primaldual interior point filter line search algorithm for large-scale nonlinear programming," Mathematical Programming, vol. 106, no. 1, pp. 25-57, 2006. 